Pacific Journal of Mathematics

DECOMPOSITIONS OF THE STONE-ČECH
COMPACTIFICATION WHICH ARE SHAPE EQUIVALENCES 


\section{DECOMPOSITIONS OF THE STONE-ČECH COMPACTIFICATION WHICH ARE SHAPE EQUIVALENCES}

\section{JAMES KeESLING}

Let $X$ be a realcompact space and $\beta X$ the Stone-Čech compactification of $X$. Let $K \subset \beta X-X$ be any nondegenerate continuum. In this paper it is shown that if $f(K)=Y$ is any map which is a shape equivalence, then $f$ is a homeomorphism. Let $X$ be realcompact and connected. Suppose that $f(\beta X)=Y$ is a continuous map which is a shape equivalence. Then it is shown that there is a compact set $K \subset Y$ such that $f^{-1}(K) \subset X$ with $f \mid \beta X-f^{-1}(K)$ a homeomorphism onto $Y-K$. In particular, if $c X$ is any compactification of $X$ and $h: \beta X \rightarrow c X$ is the natural map induced by the identity map on $X$, then if $h$ is a shape equivalence, then $h$ is a homeomorphism. Examples and applications are given.

Introduction. An important question in shape theory is: What kinds of continuous mappings give shape equivalences? That is, if $X$ and $Y$ are compact spaces and $f(X)=Y$ what conditions on $f$ would guarantee that $f$ is a shape equivalence? If $f$ is a homeomorphism or a homotopy equivalence, then $f$ is a shape equivalence. If $f$ has the property that its point inverses have trivial shape then $f$ is said to be a $C E$-map. If $f(X)=Y$ is a $C E$-map and $Y$ is finite-dimensional, then $f$ is known to be a shape equivalence. This is one of the most important types of shape equivalences. In this paper the question of when a map is a shape equivalence is studied in reverse order. We suppose that we have a map $f(X)=Y$ which is a shape equivalence and ask what this implies about the map $f$. The results we obtain are as follows. Let $X$ be a realcompact space and $K$ a continuum contained in $\beta X-$ $X$. Suppose that $f(K)=Y$ is a shape equivalence. Then we show that $f$ must be a homeomorphism. Thus maps which are shape equivalences on continua contained in $\beta X-X$ are of the simplest possible type. They are homeomorphisms. This should be contrasted with what is known about manifolds and polyhedra of dimension $\geqq 1$ which always admit nontrivial $C E$-maps which are shape equivalences.

Let $X$ be a realcompact connected space and suppose that $f(\beta X)=$ $Y$ is a shape equivalence. Then it is shown that there is a compact set $K \subset Y$ such that $f^{-1}(K) \subset X$ with $f \mid \beta X-f^{-1}(K)$ a homeomorphism onto $Y-K$. This severely restricts the kinds of maps of $\beta X$ which can be 
shape equivalences. In particular, if $c X$ is another compactification and $f: \beta X \rightarrow c X$ the natural map induced by the identity map on $X$, then if $f$ is a shape equivalence, then $f$ is a homeomorphism.

As applications of these results we show that if $X$ is realcompact and $K$ is a continuum contained in $\beta X-X$, then $K$ cannot be shape equivalent to a compact connected abelian topological group. Also $K$ cannot be torus-like. If $X$ is realcompact and connected and not compact, then $\beta X$ cannot have the shape of a compact connected abelian topological group. Also $\beta X$ cannot be torus-like. In addition a number of results in [7] are generalized.

The proofs of the above theorems make significant use of the theory of shape for compact connected abelian topological groups developed in [3], [4], [5], and [6]. Specific results will be quoted in the first section of the paper.

Preliminaries. We assume that the reader is familiar with the Stone-Čech compactification. Gillman and Jerison [1] and Walker [9] are good references. We assume a knowledge of general topology and the reader should be familiar with the terms: Lindelö, paracompact, pseudocompact, realcompact, and proper mapping. Most of the terms can be found by consulting Gillman and Jerison [1] or Isbell [2] or any advanced text in general topology. We let $H^{n}(X)$ denote the Čech cohomology of $X$ based on the numerable covers of $X$ with integer coefficients:

Let $C$ denote the category of compact spaces and continuous maps and let $S: C \rightarrow S C$ denote the shape functor. If $X$ and $Y$ are compacta, then a map $f: X \rightarrow Y$ is a shape equivalence if $S(f)$ is an equivalence in the shape category. A good reference for shape theory is Mardešić [8]. We will only be concerned with shape theory for compact spaces in this paper.

1. Continua contained in $\beta X-X$. The main result of this section is that if $X$ is realcompact and $K$ is a continuum contained in $\beta X-X$ and $f(K)=Y$ is a map which is a shape equivalence, then $f$ is a homeomorphism. We are also able to prove that $K$ cannot be movable or of trivial shape unless it is a point. This last result generalizes the main result of Keesling and Sher [7] where this was shown for continua contained in $\beta X-X$ for $X$ Lindelöf. The proofs in this paper are quite different from those in [7] and are not just a refinement of the techniques of that paper. It seems that both techniques of proof will have further applications.

We will need a number of results from [3], [4], [5], and [6] which we now state for convenient reference. 
THEOREM 1.1. (Theorem 1.1 of [4]) Let $X$ be a continuum and $x \in X$ and let $A$ be a compact connected abelian topological group. Then if $F: X \rightarrow A$ is a shape morphism, then there is a continuous map $f: X \rightarrow A$ with $f(x)=0$ and with $S(f)=F$.

THEOREM 1.2. (Theorem 1.2 of [4]) Let $X$ be a continuum and $x \in X$ and let $A$ be a compact connected abelian topological group. Then if $f$ and $g$ are continuous maps from $X$ to $A$ with $f(x)=g(x)=0$ and $S(f)=S(g)$, then $f$ and $g$ are homotopic.

The next theorem follows from the proof of Theorem 2.1 of [4].

THEOREM 1.3. Suppose that $X$ is a continuum and that $A$ is $a$ compact connected abelian topological group. Suppose that $X$ and $A$ are shape equivalent and let $F: X \rightarrow A$ be an equivalence in the shape category. Then any map $f: X \rightarrow A$ with $S(f)=F$ must be onto.

THEOREM 1.4. (Theorem 1.1 of [5]) Let $X$ be a continuum and $A$ a compact connected abelian topological group. Suppose that $h: H(A) \rightarrow H(X)$ is a homomorphism. Then there is a unique shape morphism $F: X \rightarrow A$ such that $F^{*}=h: H(A) \rightarrow H(X)$.

The last theorem we need is the following.

THEOREM 1.5. (Theorem 1.2 of [6]) Let $X$ be a torus-like continuum. Then $X$ has the shape of a compact connected abelian topological group.

We now proceed to the results in the section. We will need two preliminary lemmas.

LEMMA 1.6. Let $x \in \beta R-R$ where $R$ is the real numbers. Let $\Sigma_{a}$ be any solenoid and let $z \in \Sigma_{a}$. Then there is a continuous map $f: \beta R \rightarrow \Sigma_{a}$ such that $f(0)=0$ and $f(x)=z$.

Proof. Let $A=\left[0, \frac{1}{2}\right]+Z \subset R$ and $B=\left[\frac{1}{2}, 1\right]+Z \subset R$ where $Z$ is the integers. Now $A$ and $B$ are closed and $A \cup B=R$. Thus $x \in \operatorname{cl}_{\beta R} A$ or $x \in \operatorname{cl}_{\beta R} B$. Suppose without loss that $x \in \operatorname{cl}_{\beta R} A$. Let $\varphi: R \rightarrow \Sigma_{a}$ be a dense one-parameter subgroup. Let $r_{i} \in R$ for $i=1,2, \cdots$ be such that $\varphi\left(r_{i}\right) \rightarrow z$. Now define a map $g: R \rightarrow R$ such that $g(0)=0$ and $g\left(\left[0, \frac{1}{2}\right)+i\right)=r_{|i|}$ for $i \in Z-\{0\}$. Such a map $g$ is clearly possible. Now let $f: \beta R \rightarrow \Sigma_{a}$ be the Čech extension of the map $\varphi \circ g: R \rightarrow \Sigma_{a}$. Then $f(0)=\varphi \circ g(0)=0 \in \Sigma_{a}$ and since $x \in \operatorname{cl}_{\beta R} A, f(x)=\lim \varphi\left(r_{t}\right)=z$. 
LEMMA 1.7. Suppose that $X$ is realcompact and not compact and let $K$ be a continuum contained in $\beta X-X$. Let $x \neq y \in K$. Let $u, v \in \Sigma_{a}$ where $\Sigma_{a}$ is any solenoid. Then there is a continuous function $g: K \rightarrow \Sigma_{a}$ such that $g(x)=u$ and $g(y)=v$.

Proof. Since $X$ is realcompact, there is a continuous real-valued function $f: X \rightarrow R$ such that $\beta f: \beta X \rightarrow \beta R$ has $\beta f(x) \notin R$. Now let $q: \beta X \rightarrow[0,1]$ be a continuous map such that $q$ is 0 on a neighborhood of $y$ and 1 on a neighborhood of $x$. Then let $h: X \rightarrow R$ be defined by $h(z)=f(z) \cdot q(z)$ for all $z \in X$. Then consider $\beta h: \beta X \rightarrow \beta R$. Then $\beta h(x) \in \beta R-R$ since $h$ agrees with $f$ on a dense subset of a neighborhood of $x$ in $\beta X$. Also, $\beta h(y)=0$ since $h \equiv 0$ on a dense subset of a neighborhood of $x$ in $\beta X$. Now using Lemma 1.6, there is a map $p: \beta R \rightarrow \Sigma_{a}$ such that $p(0)=0$ and $p(\beta h(x))=u-v$. Now define $s: \Sigma_{a} \rightarrow \Sigma_{a}$ by $s(z)=z+v$ for all $z \in \Sigma_{a}$. Then let $g: \beta X \rightarrow \Sigma_{a}$ be defined by $g=s \circ p \circ \beta h$. Then $g(x)=s \circ p \circ \beta h(x)=(u-v)+v=u$ and $g(y)=s \circ p \circ \beta h(y)=s(p(0))=0+v=v$. Thus $g \mid K$ is the desired map.

We now prove a theorem which allows us to generalize several of the results of [7].

THEOREM 1.8. Suppose that $X$ is realcompact and that $K$ is a nondegenerate continuum contained in $\beta X-X$. Then $H^{1}(K)$ has the property that it contains a copy of the rational numbers as a subgroup.

Proof. Let $x, y \in K$ be distinct points. Let $\Sigma_{\omega}$ be the rational solenoid and let $v \in \Sigma_{\omega}$ be a point which is not in the same arccomponent as $0 \in \Sigma_{\omega}$. Then let $f: K \rightarrow \Sigma_{\omega}$ be a continuous function with $f(x)=0$ and $f(y)=v$ as in Lemma 1.7 .

Claim 1. The map $f$ must be onto.

Proof of Claim 1. The set $f(K)$ is a subcontinuum of $\Sigma_{\omega}$ containing 0 and $v$. However, the only proper subcontinua of a solenoid are arcs or points. Since there is no arc containing both 0 and $v, f(K)$ must be all of $\Sigma_{\omega}$. This proves Claim 1 .

Now the rational solenoid has $H^{1}\left(\Sigma_{\omega}\right)=Q$, the group of rational numbers. Consider the homomorphism $f^{*}: H^{1}\left(\Sigma_{\omega}\right) \rightarrow H^{1}(K)$. Now $H^{1}(K)$ is torsion free and thus if $f^{*}(r)=0$ for some $r \neq 0$ in $H^{1}\left(\Sigma_{\omega}\right)$, then $f^{*} \equiv 0$. We will now show that $f^{*}$ is not identically zero. It will then follow that $f^{*}(r) \neq 0$ for all nonzero $r \in H^{1}\left(\Sigma_{\omega}\right)$ and thus that $f^{*}\left(H^{1}\left(\Sigma_{\omega}\right)\right)$ is an isomorphic copy of $Q$ in $\dot{H}^{1}(K)$. Thus Theorem 1.8 will follow once we have proved Claim 2. 
Claim 2. The homomorphism $f^{*}: H^{1}\left(\Sigma_{\omega}\right) \rightarrow H^{1}(K)$ cannot be identically zero.

Proof of Claim 2. Suppose that $f^{*} \equiv 0$. Let $g: K \rightarrow \Sigma_{\omega}$ be the constant map, $g(z)=0$ for all $z \in K$. Now $g^{*}: H^{1}\left(\Sigma_{\omega}\right) \rightarrow H^{1}(K)$ has the property that $g^{*} \equiv 0$ since $g(K)$ is a point in $\Sigma_{\omega}$.

Thus we have that $f^{*} \equiv g^{*} \equiv 0$ and also $f(x)=g(x)=0$. Now by Theorem 1.4, $S(f)=S(g)$. That is, we have $S(f)=S(g)$ and $f(x)=$ $g(x)=0$. Thus by Theorem 1.2, $f$ and $g$ must be homotopic. However, this implies that there is an arc connecting $f(y)$ and $g(y)$ in $\Sigma_{\omega}$. This is a contradiction, since $g(y)=0$ and $f(y)=v$ and no arc connects 0 and $v$. This contradiction proves the claim and the proof of Theorem 1.8 is complete.

COROllary 1.9. Let $X$ be realcompact and let $K$ be a nondegenerate continuum contained in $\beta X-X$. Then $K$ cannot be movable. In particular $K$ cannot have the shape of a point.

Proof. Theorem 1.8 implies that $H^{1}(K)$ contains a copy of the rational numbers. Theorem 4.4 of [5] then implies that $K$ cannot be movable. Now $K$ cannot have trivial shape since a point is movable.

CoRollary 1.10. Let $X$ be realcompact and let $K \subset \beta X-X$ be a nondegenerate continuum. Then if $K$ has the same shape as $Y$, then $Y$ cannot be locally connected or arcwise connected.

Proof. Suppose that $K$ and $Y$ are shape equivalent. Then $H^{1}(Y)$ contains a copy of the additive group of rational numbers $Q$, since $H^{1}(Y)$ is isomorphic to $H^{1}(K)$. Thus $Y$ cannot be locally connected by Theorem 2.2 of [5]. Now we show that $Y$ cannot be arcwise connected. Let $\Sigma_{\omega}$ be the rational solenoid and let $h: H^{1}\left(\Sigma_{\omega}\right) \rightarrow H^{1}(Y)$ be an isomorphism onto the copy of $Q$ which exists by the above remarks. Let $F: Y \rightarrow \Sigma_{\omega}$ be the shape morphism given by Theorem 1.4 with $F^{*}=h: H^{1}\left(\Sigma_{\omega}\right) \rightarrow H^{1}(Y)$. Then let $f: Y \rightarrow \Sigma_{\omega}$ with $S(f)=F$ by Theorem 1.1. Now we claim that $f(Y)=\Sigma_{\omega}$. If not, then $f(Y)$ is a proper subcontinuum of $\Sigma_{\omega}$. But then $f(Y)$ is an arc or a point. But that would imply that $f^{*}: H^{1}\left(\Sigma_{\omega}\right) \rightarrow H^{1}(Y)$ is the zero homomorphism. This is a contradiction since $f^{*}=h \neq 0$. However, this now implies that $Y$ must have at least $2^{N_{0}}$ arccomponents since $f(Y)=\Sigma_{\omega}$ and $\Sigma_{\omega}$ has $2^{\boldsymbol{\kappa}_{o}}$ arccomponents.

REMARK 1.11. In [7] it was shown that for a continuum $K \subset \beta X-$ $X$ with $X$ Lindelöf, $H^{1}(K)$ contains a copy of the real numbers as a 
subgróup. The technique of proof in [7] is quite different from the proof of Theorem 1.8 given here. Thus we expect that both techniques of proof will have further applications even though the basic results are very similar.

We now prove the main theorem of this section and give some applications.

THEOREM 1.12. Suppose that $X$ is realcompact and that $K$ is a continuum contained in $\beta X-X$. Then if $f(K)=Y$ is any continuous map which is a shape equivalence, then $f$ is a homeomorphism.

Proof. Suppose that $f(K)=Y$ is a shape equivalence and that $f(x)=f(y)$ for $x \neq y$ in $K$. Let $\Sigma_{a}$ be a solenoid. Let $g: K \rightarrow \Sigma_{a}$ be a map such that $g(x)=0 \in \Sigma_{a}$ and $g(y)$ is not connected to 0 by an arc in $\Sigma_{a}$. Such a map $g$ exists by Lemma 1.7. Now we claim that if $h: Y \rightarrow \Sigma_{a}$ is any map, then $g$ is not homotopic to $h \circ f$. We will then show that this contradicts the assumption that $f$ is a shape equivalence.

Claim 1. If $h: Y \rightarrow \Sigma_{a}$ is any continuous map, then $h$ of cannot be homotopic to $g$.

Proof of Claim 1. Suppose that $h: Y \rightarrow \Sigma_{a}$ and that $h \circ f$ is homotopic to $g$. Then $h \circ f(x)=h \circ f(y)$ since $f(x)=f(y)$. Now $h \circ f(x)$ must be connected by an arc to $g(x)=0$ and $h \circ f(y)$ must be connected by an arc to $g(y)=v$. However, this implies that there is an arc from 0 to $v$ in $\Sigma_{a}$, a contradiction. Thus $h$ of cannot be homotopic to $g$ and Claim 1 is proved.

Claim 2. Since $f$ is a shape equivalence, there must be a map $h: Y \rightarrow \Sigma_{a}$ such that $h$ of is homotopic to $g$.

Proof of Claim 2. Since $f$ is a shape equivalence, $S(f)$ is an equivalence in the shape category between $K$ and $Y$. This implies that the shape morphisms from $Y$ to $\Sigma_{a}$ are in one-to-one correspondence with the set of all shape morphisms from $K$ to $\Sigma_{a}$. This correspondence is induced by composition with $S(f)$. In particular, there must be a shape morphism $F: Y \rightarrow \Sigma_{a}$ such that $F \circ S(f)=S(g)$. Now according to Theorem 1.1, there must be a map $h: Y \rightarrow \Sigma_{a}$ with $h(f(x))=0$ and with $S(h)=F$. However, then we have $S(g)=S(h) \circ S(f)=S(h \circ f)$ and $h \circ f(x)=g(x)=0$. Thus by Theorem 1.2, $h \circ f$ and $g$ must be homotopic. This proves Claim 2.

Obviously Claim 1 and Claim 2 are contradictory. This contradiction arises because we assumed $f$ to be a shape equivalence and not a homeomorphism. This completes the proof of Theorem 1.12. 
Corollary 1.13. Suppose that $X$ is realcompact and that $K \subset$ $\beta X-X$ is a nondegenerate continuum. Then $K$ cannot have the shape of a compact connected abelian topological group.

Proof. Suppose that $K$ has the shape of a compact connected abelian topological group $A$. Let $F: K \rightarrow A$ be an equivalence in the shape category. Then by Theorem 1.1 there exists a map $f: K \rightarrow A$ with $S(f)=F$, and by Theorem $1.3 f$ must have the property that $f(K)=A$. That is, $f(K)=A$ and $f$ is a shape equivalence. Thus by Theorem $1.12 f$ must be a homeomorphism. However, every compact connected abelian topological group which is not a point must contain a nontrivial one-parameter subgroup. Thus $A$ must contain an arc. However, an arc has trivial shape. That is, there must be a nondegenerate subcontinuum $C$ of $K$ having trivial shape. This is a contradiction of Corollary 1.9 since $C \subset \beta X-X$ also.

COROLlARY 1.14. Let $X$ be realcompact and let $K$ be a nondegenerate continuum contained in $\beta X-X$. Then $K$ cannot be torus-like.

Proof. Suppose that $K$ were torus-like. Then by Theorem $1.5, K$ has the shape of a compact connected abelian topological group. This contradicts Corollary 1.13 .

EXAMPLE 1.15. Example 1.6 of [7] shows that for $X$ not realcompact, the theorems and corollaries of this section may fail. The example goes as follows. Let $L$ be the long line and $Y$ any compact space. Then $\beta(L \times Y)=\left(L \cup\left\{\omega_{1}\right\}\right) \times Y$ and thus if we let $X=L \times Y$, then $\beta X-X=Y$. By appropriate choice of $Y$ we can get a space $X$ such that $\beta X-X$ is a nondegenerate continuum which has trivial shape, is a compact connected abelian topological group, or a continuum $Y$ with a nontrivial map $f(Y)=Z$ which is a shape equivalence.

2. Maps which are shape equivalences of $\beta X$. In this section we show that if $X$ is realcompact and connected and $f(\beta X)=Y$ is a shape equivalence, then $f$ is a homeomorphism on a neighborhood of $\beta X-X$. This severely restricts the kinds of maps which can be shape equivalences of $\beta X$ and allows us to draw several conclusions about such maps.

Proposition 2.1. Suppose that $X$ is realcompact and connected and that $f(\beta X)=Y$ is a shape equivalence. Then if $f\left(x_{1}\right)=f\left(x_{2}\right)=y$ with $x_{1} \neq x_{2}$, then $\left\{x_{1}, x_{2}\right\} \subset X$. 
Proof. Suppose not and let $f(\beta X)=Y$ be a shape equivalence with $f\left(x_{1}\right)=f\left(x_{2}\right)=y$ with $x_{1} \neq x_{2}$ and with $x_{1} \in \beta X-X$. Then there is a map $h: X \rightarrow R$ such that $\beta h\left(x_{1}\right) \in \beta R-R$ and $\beta h\left(x_{2}\right)=0 \in R$. If $x_{2} \in X$, then it is straightforward to construct such an $h$. If $x_{2} \notin X$, then such a map was shown to exist in the proof of Lemma 1.7. Now let $\Sigma_{a}$ be any solenoid. Let $p: R \rightarrow \Sigma_{a}$ be a map such that $p(0)=0 \in \Sigma_{a}$ and $\beta p\left(\beta h\left(x_{1}\right)\right)=v$ cannot be connected by an arc to 0 in $\Sigma_{a}$. Such a map $p$ exists by Lemma 1.6. Then let $g: \beta X \rightarrow \Sigma_{a}$ be the Cech extension of the map $p \circ h: X \rightarrow \Sigma_{a}$. Then $g\left(x_{1}\right)=v$ and $g\left(x_{2}\right)=0$.

Claim 1. If $h: Y \rightarrow \Sigma_{a}$ is any continuous map, then $h \circ f$ cannot be homotopic to $g$.

Proof of Claim 1. This is the same as the proof of Claim 1 in the proof of Theorem 1.12.

Claim 2. Since $f$ is a shape equivalence, there must be a map $h: Y \rightarrow \Sigma_{a}$ such that $h$ of is homotopic to $g$.

Proof of Claim 2. This is the same as the proof of Claim 2 in the proof of Theorem 1.12.

Clearly Claim 1 and Claim 2 are contradictory. Thus we cannot have the situation assumed and Proposition 2.1 must be true.

COROLLARY 2.2. Let $X$ be realcompact and connected and suppose that $f(\beta X)=Y$ is a shape equivalence. Then for any $y \in Y$ with $f^{-1}(y)$ nondegenerate $f^{-1}(y) \subset X$. Thus $f \mid X$ must be a proper map onto $f(X)$ and $f \mid \beta X-X$ must be a homeomorphism with $f(X) \cap f(\beta X-X)=\varnothing$.

The next theorem, which is the main result of this section, shows that not only is it true that $f \mid \beta X-X$ is a homeomorphism, but $f$ restricted to a neighborhood of $\beta X-X$ must be a homeomorphism.

THEOREM 2.3. Let $X$ be realcompact and connected and suppose that $f(\beta X)=Y$ is a shape equivalence. Let $A=\left\{y \in Y \mid f^{-1}(y)\right.$ is not a single point $\}$. Then $f^{-1}(A) \subset X$ and $\operatorname{cl}_{X} f^{-1}(A)$ is compact.

Proof. The above corollary shows that $f^{-1}(A) \subset X$. We now set about to show that $\operatorname{cl}_{X} f^{-1}(A)$ is compact. The proof will be by contradiction. Suppose that $B=f^{-1}(A)$ does not have compact closure in $X$. Then $\left(\operatorname{cl}_{\beta X} B\right) \cap(\beta X-X) \neq \varnothing$. Let $x \in \operatorname{cl}_{\beta X} B$ with $x \notin X$. Since $X$ is realcompact, there must be a map $g: X \rightarrow R$ with $\beta g(x) \in \beta R$ $R$. Let $b_{1} \in B$ be a sequence with $\left|g\left(b_{1}\right)\right| \rightarrow \infty$. Such a sequence exists since $x \in \operatorname{cl}_{\beta X} B$. Now for each $y \in Y, f^{-1}(y)$ is compact. Thus $f^{-1}(y) \cap$ 
$\left\{b_{1}\right\}$ is finite for each $y \in Y$. Thus, without any loss, we may assume that $f\left(b_{1}\right) \neq f\left(b_{1}\right)$ for $i \neq j$. Since $b_{1} \in B=f^{-1}(A)$ for each $i$, there must be a $c_{\imath} \in B$ with $c_{\imath} \neq b_{\imath}$ with $f\left(c_{\imath}\right)=f\left(b_{l}\right)$. Let $\left\{c_{l}\right\}$ be a sequence of such points.

Claim 1. It must be that $\operatorname{cl}_{\beta X}\left\{b_{1}\right\} \cap \mathrm{cl}_{\beta X}\left\{c_{1}\right\}=\varnothing$.

Proof of Claim 1. By Corollary 2.2, $f \mid X$ is a proper map onto $f(X)$. Since $\left\{b_{1}\right\}$ is a closed discrete subset of $X, f\left(\left\{b_{t}\right\}\right)$ must be closed and discrete in $f(X)$. But $\left\{c_{l}\right\}$ is obtained by choosing one point out of $f^{-1}\left(f\left(b_{1}\right)\right)$ for each $i$. Thus, $\left\{c_{i}\right\}$ must also be a closed discrete subset of $X$. Now since $\left|g\left(b_{1}\right)\right| \rightarrow \infty$, the set $\left\{b_{1}\right\}$ must be $C$-embedded in $X$. Thus there is a continuous map $k: X \rightarrow R$ with $k\left(b_{i}\right)=i$ for $i=1,2, \cdots$. Let $U_{i}$ be an open set in $X$ containing $b_{i}$ such that $U_{i} \subset k^{-1}\left(i-\frac{1}{4}, i+\frac{1}{4}\right)$ for each $i$ and $U_{i} \cap\left\{c_{l}\right\}=\varnothing$ for each $i$. Then $\left\{U_{1}\right\}$ is a disjoint discrete collection in $X$. Let $h_{1}: \bar{U}_{1} \rightarrow[0,1]$ be defined so that $h_{1}\left(b_{1}\right)=1$ and $h_{1}\left(\bar{U}_{1}-U_{t}\right) \equiv 0$. Define $h: X \rightarrow[0,1]$ by $h \mid \bar{U}_{\imath}=h_{\mathrm{t}}$ and $h(x)=0$ for $x \notin \bigcup_{i=1}^{\infty} U_{i}$. Then $h \mid\left\{b_{l}\right\} \equiv 1$ and $h \mid\left\{c_{i}\right\} \equiv 0$. Thus $\mathrm{cl}_{\beta X}\left\{b_{1}\right\} \subset \beta h^{-1}(1)$ and $\mathrm{cl}_{\beta X}\left\{c_{1}\right\} \subset \beta h^{-1}(0)$. Thus $\mathrm{cl}_{\beta X}\left\{b_{1}\right\} \cap \mathrm{cl}_{\beta X}\left\{c_{1}\right\}=\varnothing$ and Claim 1 is established.

Claim 2. There is a point $p \in \operatorname{cl}_{\beta X}\left\{b_{1}\right\}-X$ and a point $q \in \operatorname{cl}_{\beta X}\left\{c_{\imath}\right\}$ such that $f(p)=f(q)$.

Proof of Claim 2. Let $p \in \mathrm{cl}_{\beta X}\left\{b_{1}\right\}-X$. Let $\left\{b_{t_{\alpha}}\right\}$ be a subnet of the sequence $\left\{b_{1}\right\}$ such that $b_{1 a} \rightarrow p$. Let $\left\{c_{1 \beta}\right\}$ be a subnet of the net $\left\{c_{l_{\alpha}}\right\}$ such that $\left\{c_{t_{\beta}}\right\}$ converges to some point $q \in \mathrm{cl}_{\beta X}\left\{c_{1}\right\}$. Then $b_{t \beta} \rightarrow p$ and $c_{1 \beta} \rightarrow q$. Thus $f\left(b_{1 \beta}\right) \rightarrow f(p)$ and $f\left(c_{1 \beta}\right) \rightarrow f(q)$. However, $f\left(b_{1}\right)=f\left(c_{1}\right)$ for all $i$ so that these two nets, $\left\{f\left(b_{1 \beta}\right)\right\}$ and $\left\{f\left(c_{t \beta}\right)\right\}$ are identical. Thus $f(p)=f(q)$. This proves Claim 2 .

The points $p$ and $q$ given by Claim 2 must have the property that $p \neq q$ by Claim 1. However, this then contradicts Proposition 2.1. This completes the proof of Theorem 2.3.

We conclude this section by giving several applications of Theorem 2.3 and some examples.

Corollary 2.4. Suppose that $X$ is connected and realcompact and suppose that $c X$ is a compactification of $X$. Let $f: \beta X \rightarrow c X$ be the natural map induced by the identity map on $X$. Then if $f$ is a shape equivalence, then $f$ is a homeomorphism.

Proof. Suppose that $f$ is a shape equivalence. Now $X$ is dense in $c X$ and consequently $f(\beta X)=c X$. According to Theorem 2.3 if $A=$ 
$\left\{y \in c X \mid f^{-1}(y)\right.$ is not a single point $\}$, then $f^{-1}(A) \subset X$. However, by assumption $f \mid X$ is the identity map. Thus $A=\varnothing$ and $f$ is a homeomorphism.

EXAMPLE 2.5. In this example we will show that we may have compactifications $d X$ and $c X$ and a natural map $f: d X \rightarrow c X$ which is a shape equivalence and not a homeomorphism. Let $n \geqq 2$ and let $I$ be a tame arc in $S^{n}$. Then $R^{n}$ is homeomorphic to $S^{n}-I$. Also $S^{n}$ is homeomorphic to the one-point compactification of $R^{n}$. Let the first compactification be denoted $d X$ and the second $c X$ with $X=R^{n}$. Let $f: d X \rightarrow c X$ be defined by $f(I)=\infty$ and $f \mid R^{n}$ is a homeomorphism. Then $f$ is a $C E$-map, and hence a shape equivalence. But $f$ is not a homeomorphism.

EXAMPLE 2.6. Let $L$ be the long line and $I$ be the unit interval. Then let $X=L \times I$. Then $\beta X=L \cup\left\{\omega_{1}\right\} \times I$ and this space has trivial shape. Let $f(\beta X)=\{p t\}$. Then $f$ is a shape equivalence. Now $X$ is not realcompact. This example shows that 2.1, 2.2 , and 2.3 are not true if the assumption that $X$ is realcompact is dropped.

The last result of this section is that $\beta X$ cannot have the shape of a compact connected abelian topological group.

THEOREM 2.7. Let $X$ be a connected realcompact space which is not compact. Then $\beta X$ cannot have the shape of a compact connected abelian topological group.

Proof. Suppose that $\beta X$ has the same shape as the compact connected abelian topological group $A$ and let $f(\beta X)=A$ be a map which is a shape equivalence (see the proof of Corollary 1.13). Let $B \subset A$ be a compact set containing all the elements $y \in A$ with $f^{-1}(y)$ not a single point and with $f^{-1}(B) \subset X$. Such a set $B$ exists by Theorem 2.3. Let $x \in f(\beta X-X)$. Then there is an arc $I$ containing $x$ with $I \subset A-B$. Now we claim that such an arc cannot exist. Note that $I \cap f(\beta X-X)$ is totally disconnected, otherwise $\beta X-X$ would contain a nondegenerate arc which it does not. Thus $X \cap f^{-1}(I)$ is dense in $f^{-1}(I)$. et $h: X \rightarrow R$ be a continuous function such that $\beta h: \beta X \rightarrow \beta R$ takes $y=f^{-1}(x)$ to a point in $\beta R-R$. But then $\beta h\left(f^{-1}\right)$ restricted to some subarc of $I$ determines a path in $\beta R$ joining some point of $R$ to $\beta h(y) \in \beta R-R$. No such path exists in $\beta R$ and consequently no such arc can exist in $A-B$. That is, $B=A$ and the map $f$ cannot be a shape 
equivalence because we have a contradiction to Theorem 2.3. This proves Theorem 2.7 .

3. Realcompact spaces. We conclude the paper with a remark about realcompact spaces. It is known that a metric space is realcompact if it is of nonmeasurable cardinality $[1,15.24$, p. 232]. Also every Lindelöf space is realcompact $[1,8.2$, p. 115]. We now give a simple proof that every paracompact space of nonmeasurable cardinality is also realcompact.

THEOREM 3.1. Every paracompact space of nonmeasurable cardinality is realcompact.

Proof. Assume that $X$ is a paracompact space of nonmeasurable cardinality. Let $x \in \beta X-X$. We must construct a continuous function $f: X \rightarrow R$ such that the extension $\beta f$ takes $x$ to a point of $\beta R-R$. For each $y \in X$ there is an open set $U_{y}$ with $y \in U_{y}$ and $\operatorname{cl}_{\beta X} U_{y}$ not containing the point $x$. For each $y \in X$, let $U_{y}$ be chosen and let $\mathcal{u}=\left\{U_{y}: y \in X\right\}$.

Now every paracompact space is a uniform space with each open cover a member of the uniformity. Thus by [2, Theorem 14, p. 7] there is a continuous map $g(X)=M$ with $M$ a metric space such that every subset $S$ of $M$ with diameter less than 1 has the property that $g^{-1}(S) \subset U_{y}$ for some $y \in X$.

Claim. If $\beta g: \beta X \rightarrow \beta M$ is the Čech extension of $g$, then $\beta g(x) \notin M$.

Proof of Claim. Suppose that $\beta g(x) \in M$. Let $S$ be the $\frac{1}{4}$-ball about $\beta g(x)$. Then there is a $y \in X$ with $g^{-1}(S) \subset U_{y}$. Let $h: M \rightarrow[0,1]$ be such that $h(\beta g(x))=1$ and $h \equiv 0$ on the complement of $S$. Then $h \circ g: X \rightarrow[0,1]$ has the property that $h \circ g$ vanishes outside $U_{y}$. Thus $\beta(h \circ g)$ vanishes outside $\operatorname{cl}_{\beta x} U_{y}$. Thus $\beta(h \circ g)(x)=$ 0 . However, this contradicts the fact that $\beta(h \circ g)(x)=\beta h \circ \beta g(x)=$ $h(\beta g(x))=1$. This proves the claim.

Now $M$ must be of nonmeasurable cardinality since $X$ is. Thus $M$ is realcompact. Thus there must be a map $h: M \rightarrow R$ such that $\beta h(\beta g(x)) \in \beta R-R$. But then $h \circ g: X \rightarrow R$ is a map which has the property that $\beta(h \circ g)(x) \in \beta R-R$. Thus $X$ must be realcompact. This proves Theorem 3.1. 


\section{REFERENCES}

1. L. Gillman and M. Jerison, Rings of Continuous Functions, Springer-Verlag, New York, 1976.

2. J. Isbell, Uniform Spaces, Amer. Math. Soc., Providence, 1964.

3. J. Keesling, Shape theory and compact connected abelian topological groups, Trans. Amer. Math. Soc., 194 (1974), 349-358.

4. - Continuous functions induced by shape morphisms, Proc. Amer. Math. Soc., 41 (1973), 315-320.

5. _- An algebraic property of the Čech cohomology groups which prevents local connectivity and movability, Trans. Amer. Math. Soc., 190 (1974), 151-162.

6. - On the shape of torus-like continua and compact connected topological groups, Proc. Amer. Math. Soc., 40 (1973), 297-302.

7. J. Keesling and R. B. Sher, Shape properties of the Stone-Čech compactification, Gen. Top. and Appl. (to appear).

8. S. Mardešić, Shapes for topological spaces, Gen. Topology and Appl., 3 (1973), 265-282.

9. R. C. Walker, The Stone-Čech Compactification, Springer-Verlag, New York, 1974.

Received February 15, 1977 and in revised form May 11, 1977.

UNIVERSITY OF FLORIDA

GAINESVILLE, FL 32611 


\section{PACIFIC JOURNAL OF MATHEMATICS}

\section{EDITORS}

RICHARD ARENS (Managing Editor)

University of California

Los Angeles, CA 90024

R. A. BeAumont

University of Washington

Seattle, WA 98105

C. C. MOORE

University of California

Berkeley, CA 94720
J. DUGUNDJI

Department of Mathematics

University of Southern California

Los Angeles, CA 90007

R. FINN AND J. MILGRAM

Stanford University

Stanford, CA 94305

\section{ASSOCIATE EDITORS}
E. F. BECKENBACH
B. H. NEUMANN
F. WOLF
K. YoshidA

\section{SUPPORTING INSTITUTIONS}

UNIVERSITY OF BRITISH COLUMBIA

UNIVERSITY OF SOUTHERN CALIFORNIA

CALIFORNIA INSTITUTE OF TECHNOLOGY

STANFORD UNIVERSITY

UNIVERSITY OF CALIFORNIA

UNIVERSITY OF HAWAII

MONTANA STATE UNIVERSITY

UNIVERSITY OF TOKYO

UNIVERSITY OF NEVADA

UNIVERSITY OF UTAH

NEW MEXICO STATE UNIVERSITY

OREGON STATE UNIVERSITY

UNIVERSITY OF OREGON

OSAKA UNIVERSITY

WASHINGTON STATE UNIVERSITY

UNIVERSITY OF WASHINGTON

AMERICAN MATHEMATICAL SOCIETY

The Supporting Institutions listed above contribute to the cost of publication of this Journal, but they are not owners or publishers and have no responsibility for its contents or policies.

Mathematical papers intended for publication in the Pacific Journal of Mathematics should be in typed form or offset-reproduced (not dittoed), double spaced with large margins. Underline Greek letters in red, German in green, and script in blue. The first paragraph or two must be capable of being used separately as a synopsis of the entire paper. Items of the bibliography should not be cited there unless absolutely necessary, in which case they must be identified by author and Journal, rather than by item number. Manuscripts, in duplicate, may be sent to any one of the four editors. Please classify according to the scheme of Math. Reviews, Index to Vol. 39. All other communications should be addressed to the managing editor, or Elaine Barth, University of California, Los Angeles, California, 90024.

100 reprints are provided free for each article, only if page charges have been substantially paid. Additional copies may be obtained at cost in multiples of 50 .

The Pacific Journal of Mathematics is issued monthly as of January 1966. Regular subscription rate: $\$ 72.00$ a year (6 Vols., 12 issues). Special rate: $\$ 36.00$ a year to individual members of supporting institutions.

Subscriptions, orders for numbers issued in the last three calendar years, and changes of address should be sent to Pacific Journal of Mathematics, 103 Highland Boulevard, Berkeley, California, 94708.

PUBLISHED BY PACIFIC JOURNAL OF MATHEMATICS, A NON-PROFIT CORPORATION

Printed at Jerusalem Academic Press, POB 2390, Jerusalem, Israel. 


\section{Pacific Journal of Mathematics}

\section{Vol. 75, No. $2 \quad$ October, 1978}

Susan Jane Zimmerman Andima and W. J. Thron, Order-induced

topological properties ................................... 297

Gregory Wade Bell, Cohomology of degree 1 and 2 of the Suzuki groups . . 319

Richard Body and Roy Rene Douglas, Rational homotopy and unique

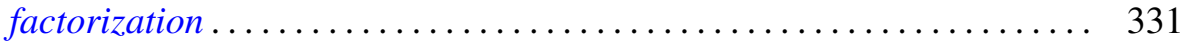

Frank Lewis Capobianco, Fixed sets of involutions ................. 339

L. Carlitz, Some theorems on generalized Dedekind-Rademacher sums .... 347

Mary Rodriguez Embry and Alan Leslie Lambert, The structure of a special class of weighted translation semigroups .....................

Steve Ferry, Strongly regular mappings with compact ANR fibers are

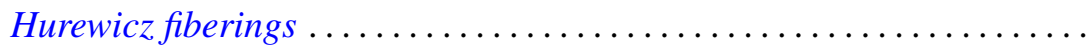

Ivan Filippenko and Marvin David Marcus, On the unitary invariance of the

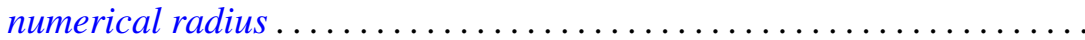

$\mathrm{H}$. Groemer, On the extension of additive functionals on classes of convex

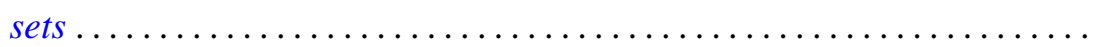

Rita Hall, On the cohomology of Kuga's fiber variety ............... 411

H. B. Hamilton, Congruences on $\mathrm{N}$-semigroups ................. 423

Manfred Herrmann and Rolf Schmidt, Regular sequences and lifting

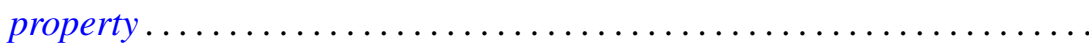

James Edgar Keesling, Decompositions of the Stone-Čech compactification which are shape equivalences .....................

Michael Jay Klass and Lawrence Edward Myers, On stopping rules and the expected supremum of $S_{n} / T_{n}$

Ronald Charles Linton, $\lambda$-large subgroups of $C_{\lambda}$-groups

William Owen Murray, IV and L. Bruce Treybig, Triangulations with the free cell property ............................

Louis Jackson Ratliff, Jr., Polynomial rings and $H_{i}$-local rings ...

Michael Rich, On alternate rings and their attached Jordan rings....

Gary Sampson and H. Tuy, Fourier transforms and their Lipschitz

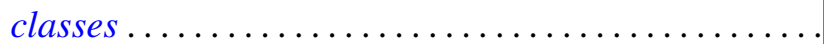

Helga Schirmer, Effluent and noneffluent fixed points on dendrites ...

Daniel Byron Shapiro, Intersections of the space of skew-symmetric maps

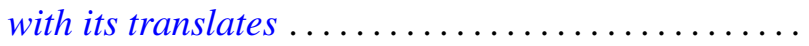

Edwin Spanier, Tautness for Alexander-Spanier cohomology ...

Alan Stein and Ivan Ernest Stux, A mean value theorem for binary digits ...

Franklin D. Tall, Normal subspaces of the density topology . .

William Yslas Vélez, Prime ideal decomposition in $F\left(\mu^{1 / p}\right) \ldots$ 